\title{
Mehrheitsregel überdacht - theoriepolitisch reaktionär, aber ideengeschichtlich instruktiv
}

\section{Flaig, Egon: Die Mehrheitsentscheidung. Entstehung und kulturelle Dynamik, Ferdinand Schö- ningh, Paderborn 2013, 628 Seiten, € 58,-.}

Es mangelt heute nicht an politikwissenschaftlicher Fachliteratur zum Thema Mehrheitsentscheidung. Zu diesem Thema etwas Neues beizutragen, ist kein leichtes Unterfangen. Und schon aus diesem Grund liest man das neue über 600 Seiten lange Buch des Rostocker Althistorikers Egon Flaig nicht ohne gespannte Erwartung, verspricht er den Lesern doch gleich zum Auftakt, dass erst die von ihm geleistete umfassende Rückbesinnung auf die verschiedenen historischen Entstehungskontexte der Mehrheitsregel ihren rationalen Kern auch für unsere heutige Zeit freilege.

Flaig zufolge verdient nur eine einzige Entscheidungsregel das Prädikat „demokratisch“: die Mehrheit. Die Begründung dafür lautet, dass das Mehrheitsprinzip die einzige Entscheidungsregel sei, in der politische Gleichheit zum Ausdruck gelangt. Wann immer ihre Durchschlagskraft durch Kompromissarrangements, institutionalisierte Veto-Positionen, Quoren oder Konkordanzsysteme kanalisiert werde, handelt es sich Flaig zufolge um nicht-demokratische politische Praktiken. Diesem Verdikt verfällt auch der moderne Parlamentarismus - wobei man als Rezensent dem Buch einen Gefallen tut, wenn man für diese zwischen Populismus und Uninformiertheit changierenden Passagen einen Mantel des Schweigens gefunden hat, der groß genug ist, dass darunter auch gleich noch seine zum Teil abstrusen Kommentare zu den Themen Atomenergie und den ökologisch motivierten Protestbewegungen oder seine Habermas-Phobie Platz finden.

Wenn es tatsächlich das primäre Ziel von Egon Flaig gewesen sein sollte, den Lesern seines Buches überzeugende Gründe für eine normative Privilegierung der Mehrheitsregel zu präsentieren, die zugleich in einer aktuellen Reformempfehlung münden soll, dann ist er daran grandios gescheitert. Denn den altbekannten Einwänden gegen die unbedingte Geltung der Mehrheitsregel seit Tocqueville, Benjamin Constant und John Stuart Mill bis heute vermag er nicht mehr entgegenzuhalten als die gebetsmühlenhafte Wiederholung der These, dass nur bei der Mehrheitsregel die politische Gleichheit konsequent zum Zuge kommt. Was aber - so fragten nicht nur die aufgebrachten Demonstranten des zivilgesellschaftlichen Protestes im Juni 2013 auf dem Taksim-Platz in Istanbul - wenn der türkische Ministerpräsident Erdogan den Protestierenden im Namen der Mehrheit die Ausübung dieser gleichen politischen Rechte gewaltsam streitig macht? Genau für solche (nicht seltenen) Fälle wurden in modernen liberalen Demokratien institutionelle Sicherungen des Minderheitenschutzes gegenüber dem Vorrang der Mehrheitsregel eingebaut. Diese Sicherungen mögen an der einen Stelle zu stark sein (wie meines Erachtens bei der Eigentumsgarantie) oder an anderer Stelle missbräuchlich zum Schutze gesellschaftlicher Herrschaftsinteressen (dito) eingesetzt werden - derartige Mängel machen die von Liberalen forcierte Problematisierung der Mehrheitsregel deswegen noch lange nicht zu Makulatur. In dieser Hinsicht ist das sich durch das gesamte Buch ziehende engagierte Plädoyer Flaigs für den normativen Vorrang der Mehrheitsregel politikwissenschaftlich naiv und theoriepolitisch reaktionär.

Und dennoch hat Egon Flaig ein überaus lesenswertes Buch zum Thema Mehrheitsregel vorgelegt. Er bezeichnet seinen Ansatz als „reflexive Anthropologie“ mit einem globalhistorischen Fragehorizont. Was darunter zu verstehen ist, erfährt man am besten in den Kapiteln, 
in denen Flaig unterschiedliche, von der bisherigen politikwissenschaftlichen Forschung kaum wahrgenommene Entstehungen der Mehrheitsregel ins Licht der Aufmerksamkeit rückt. Eine der historischen Pointen des Buches besteht im Nachweis, dass „das Rad“ namens Mehrheitsprinzip unter völlig unterschiedlichen gesellschaftlichen und politischen Randbedingungen mehrfach „neu erfunden“ worden ist: beispielsweise in verschiedenen Republiken in Nordindien zwischen 600 und 300 vor Beginn der christlichen Zeitangabe; im vorexilischen Judentum; in Dorfgemeinden auf Island im 9. Jahrhundert; in japanischen Klöstern im 13. Jahrhundert; oder einige Jahrhunderte später auf Stammesversammlungen in Samoa. Es gab also nicht den einen Ursprung, von dem aus das Mehrheitsprinzip per Kulturtransfer diffundiert ist, sondern mehrfache originäre Emergenzen.

Besonders ausführlich schildert der Althistoriker Flaig die Genesis und das Funktionieren des Mehrheitsprinzips in den griechischen Stadt- und Flächenstaaten der Antike seit dem 8. Jahrhundert v. Chr., denn hier wurde für die Mehrheitsregel von ihren Protagonisten das demokratische Gleichheitsideal explizit in Anschlag gebracht. Und Athen dient ihm auch als Paradebeispiel für seine These, dass einer Mehrheitsentscheidung immer eine kontroverse Debatte vorausgehen muss und somit Dissens und Deliberation kultiviert. Ergänzt werden die Kapitel und Abschnitte zum antiken Griechenland, die sich auf insgesamt gut die Hälfte des Buchumfanges verteilen, um bemerkenswerte Ausführungen zum Schicksal der Mehrheitsregel im republikanischen und kaiserlichen Rom sowie in der Entscheidungspraxis der katholischen Kirche.

Flaig ist ein produktiver Revisionist der Historiografie der Mehrheitsregel. In normativer Hinsicht bedeutsam ist in diesem Zusammenhang vor allem die am historischen Material überzeugend vorgeführte Zurückweisung der unter anderem von Otto von Gierke (1873) und Georg Simmel (1908) formulierten und in der Literatur vielfach heute noch dominierenden These, dass die Anerkennung des Majoritätsprinzips ursprünglich nichts weiter gewesen sei als die Verwandlung eines gewaltsamen Zwanges in einen Rechtszwang, bei dem also gleichsam das Zählen der Fäuste die Notwendigkeit eines tatsächlichen Faustkampfes ersetzt habe.

In den Abschnitten zur griechischen und römischen Antike läuft Egon Flaig zu deskriptiver und analytischer Hochform auf, und man kann sich in seinen Lesefrüchten mit ihren zahlreichen Beispielen, Textbelegen, Anekdoten und Fußnotenscharmützeln geradezu genüsslich verlieren. Aber auch nach einer plastischen Schilderung der Fehlentscheidung der Athener, den Sizilien-Feldzug zu beginnen oder einer eingestreuten Funktionsanalyse des Ostrakismos gelingt es Flaig immer wieder, den Leser auf die Bahn der ihm wichtigen systematischen Fragen zurückzuleiten: Worin bestehen jeweils die konkreten politischen und gesellschaftlichen Voraussetzungen der Mehrheitsregel? Welche Verpflichtungen werden von Mehrheitsentscheidungen erzeugt? In welchem Verhältnis stehen vorgängige Diskussion und Abstimmung zueinander? Wann funktioniert die Mehrheitsregel nicht? Welche Abstimmungsformen (öffentlich oder geheim) passen am besten zu ihr? Welche Folgen hat sie im Hinblick auf die Akustik für Architektur und Sitzordnungen? Welche Materialien finden für die Abstimmungspraxis Verwendung? Welche gesellschaftlichen Dynamiken werden durch die Anwendung der Mehrheitsregel freigesetzt? Welche spezifischen Probleme erzeugt sie? Welche nicht-intendierten Konsequenzen werden durch sie ausgelöst? Welche Risiken birgt die Mehrheitsregel, und wie wurden die Gefahren beispielsweise in Athen erfolgreich institutionell eingehegt? Worin bestehen die Vor- und Nachteile des Abstimmens nach der Mehrheitsregel im Vergleich mit dem Konsensprinzip, dem Losen und anderen Entscheidungsverfahren? 
Um diese Fragen zu beantworten, hat Flaig nicht nur das historische Material bemüht, sondern auch Debatten aus der modernen ökonomischen Entscheidungstheorie, Theorien der deliberativen Demokratie und Thesen von John Rawls in seine Überlegungen mit eingeflochten. Als Stärke des Buches ist anzusehen, dass Flaig auf die aufgelisteten Fragen keine einheitlichen Antworten geben kann und will; vielmehr belässt er es bei dem historisch informierten Facettenreichtum der Voraussetzungen, Funktionsweisen und Dynamiken der Mehrheitsregel. Sein Buch lädt den Leser dazu ein, zu eigenen Antworten auf die aufgeworfenen Fragen zu gelangen.

Je mehr man sich in den Band vertieft, desto stärker gewinnt man im Übrigen den Eindruck, dass sich die Anhängerschaft des Verfassers für die Mehrheitsregel möglicherweise nicht allein dem wortreich zur Schau gestellten politischen Egalitarismus der Athener (bei einer zuweilen befremdlich anmutenden souveränen Ignoranz gegenüber dem radikalen Anti-Egalitarismus in der sozialen Sphäre) verdankt. Denn zum einen gehört nach Meinung von Flaig nichts Geringeres als die Entstehung der Wissenschaft zu einer der notwendigen Folgen der Mehrheitsregel in Griechenland. Und zum anderen hebt er mehrfach positiv hervor, wie die mit der Mehrheitsregel verbundene Senkung der politischen Entscheidungskosten Athen an Effizienz und politischer Schlag- und Expansionskraft gewinnen ließ.

Wie dem auch sei - der Autor hat in der Einleitung nicht zu viel versprochen: Das Buch bietet viel bislang nicht zu Lesendes und Anregendes zum Thema Mehrheitsregel und sei deshalb zur Lektüre unbedingt empfohlen.

Hubertus Buchstein

\section{Regieren in Theorie, Praxis und Vergleich - gelungene Festschrift}

Egner, Björn, Michael Haus und Georgios Terizakis (Hrsg.): Regieren. Festschrift für Hubert Heinelt, VS Verlag für Sozialwissenschaften, Wiesbaden 2012, 563 Seiten, € 69,95.

Regieren ist ein komplexer, interessengeleiteter, langsamer und enttäuschungsanfälliger Prozess. „Ungenügender Erfolg“, so Friedbert W. Rüb, ist eine unvermeidbare Begleiterscheinung moderner Staatstätigkeit (S. 131). Werner Jann hat es Mitte der 1990er Jahre konzise formuliert: „Regierungen steuern nicht, sie intervenieren.“

Fünf Dimensionen legen sich wie Jahresringe um den Begriff des Regierens: In klassischer Perspektive geht Regieren von der Steuerungsfähigkeit des politisch-administrativen Systems aus. Angesichts steigender gesellschaftlicher Komplexität stehen zweitens verhandlungsdemokratische Arrangements, die auch von Regierungen unterstützt werden. Drittens - und eigentlich an zentraler Stelle - geht es beim Regieren um „policy making“, mithin um die zyklische Abfolge einzelner Schritte im Politikprozess. Heute werden viertens westliche Regierungssysteme vielfach mit dem Etikett der Postdemokratie versehen, das eine ganze Bandbreite an Defiziten auf den Begriff zu bringen versucht. Und fünftens firmiert modernes Regieren unter Governance und soll dabei komplexe Mischformen aus staatlicher Regulierung und gesellschaftlicher Selbststeuerung bedeuten.

Alle diese genannten Aspekte werden im vorliegenden Sammelband aus unterschiedlichen Perspektiven angesprochen. Konzipiert ist das Buch als Festschrift für Hubert Heinelt, der 